\title{
Congenital cytomegalovirus infection in newborn infants of mothers infected before pregnancy*
}

\author{
KURT SCHOPFER, EDGAR LAUBER, AND ULRICH KRECH \\ From the Institute of Medical Microbiology, and Children's Hospital, St Gallen, Switzerland; and \\ Nestlé Foundation, Abidjan, Ivory Coast
}

SUMMARY The rate of congenital cytomegalovirus (CMV) infection was studied in newborn infants in an African population in which all adults had experienced primary CMV infection during childhood. Viruria within the first 12 hours after delivery was taken as evidence of prenatal CMV infection. 28 of 2032 newborn infants examined had viruria, giving a rate of $1.4 \%$ congenital CMV infection. The presence of maternal serum antibody therefore appears not to protect the fetus from intrauterine infection. Either reactivation of latent maternal CMV infection or recurrence of infection during pregnancy despite the presence of serum antibodies may explain these findings. Whether the long-term effects of CMV infection acquired in utero differ in cases of primary maternal infection from those due to reactivated or recurrent infection in seropositive mothers, remains undecided. Thus, the value of a live CMV vaccine to prevent prenatal CMV infection may be questioned.

Cytomegalovirus (CMV) infection is the most common congenital infection (Klein et al., 1976). It has been assumed that the virus gains access to the fetus mainly during primary infection of the pregnant woman (Stern and Tucker, 1973). However, there are also reports of prenatal CMV infection in newborn infants of mothers who had experienced primary CMV infection before pregnancy (Embil et al., 1970; Krech et al., 1971b; Stagno et al., 1973). Thus the role of maternal immunity in protecting the fetus from being infected in utero is not understood. Nor is the significance of prenatal CMV infection in newborn infants of mothers who are seropositive before pregnancy yet established. Therefore, the study of the rate of congenital CMV infection in a population in which all adults show evidence of past CMV infection may lead to a better understanding of host immunity to CMV. We have already surveyed an African population to determine the prevalence of CMV infection as measured by the presence of serum antibodies (Krech, 1973). Congenital infection was assessed by the isolation of CMV from infants within the first hours of delivery.

Received 8 December, 1977

*This study represents phase three within the CMV investigation programme of WHO (Krech, 1973)

\section{Materials and methods}

Five hundred male blood donors attending the Centre de Transfusion in Abidjan, Ivory Coast, and 338 apparently healthy children aged between 4 and 16 years were serologically screened for complement-fixing (CF) CMV antibodies. Blood specimens from these children were obtained during routine laboratory examinations for parasitic diseases. 2155 urine samples from babies were collected into urine bags within the first 12 hours of delivery. From the babies screened for viruria 1038 cord blood samples were available for serological testing. Head circumference and weight of all babies were recorded, and the age and the number of pregnancies of their mothers. The mothers had been admitted to the hospital just before delivery and most of them left the same day with the infant; unfortunately, no other controls could be incluaded.

Serological studies. All sera were stored at $-20^{\circ} \mathrm{C}$ until tested for CF antibodies against CMV antigen. Twofold dilutions of heat-inactivated serum were assayed in microtitre plates in accordance with a method using a glycine-extracted tissue culture antigen (Krech et al., 1971a). An antibody titre of 1:4 or greater was considered as evidence of past CMV infection. 
Virological studies. Urine samples were mixed with an equal volume of a transport medium (35\% sorbitol in Eagle's minimal essential medium enriched with $2 \%$ fetal calf serum, amino-acids, and vitamins) penicillin, streptomycin, gentamicin, kanamycin, and mycostatin were added. These samples were stored for up to 7 days at $4^{\circ} \mathrm{C}$ until shipped by air from Abidjan to Switzerland direct without refrigeration; they arrived at the institute within 3 or 4 days. Human fibroblast monolayers were inoculated with aliquots of urine (Krech et al., 1971a). The cultures were kept for at least 7 weeks. For further identification the infected cells were observed by indirect immunofluorescence staining using a human serum with a high CF CMV antibody titre $(1: 240)$ and containing no antibodies against herpes viruses or adenoviruses when examined by immunofluorescence.

\section{Results}

Serological studies. 1876 sera were available. Of these $1395(74 \cdot 4 \%)$ could be used for assaying CF CMV antibodies. 481 samples were anticomplementary. The prevalence of CMV antibodies in the different population groups is shown in the Table. At 14 years, the age of the youngest pregnant woman in this study, $99.7 \%$ of the subjects tested were seropositive for CF CMV antibodies, so that virtually all women had experienced primary CMV infection before pregnancy. In only one cord blood out of 702 were CMV antibodies absent. There was an unequal distribution of CMV antibody titres in the serum samples of the different population groups tested (Fig. 1). The highest titres were found in cord blood, the lowest in the children aged between 4 and 8 years.

Virological studies. Among 2155 urine samples collected $123(5 \cdot 7 \%)$ were either contaminated or otherwise toxic to human fibroblasts. CMV was isolated from 28 samples giving a rate of $1.4 \%$ prenatal CMV infection. Cytopathogenic changes

Table CF CMV antibodies in different population groups

\begin{tabular}{lll}
\hline & $n^{*}$ & $\begin{array}{l}\text { Seropositive } \\
\text { No. (\%) }\end{array}$ \\
\hline Children & 292 & $269(92)$ \\
4-8 years & 158 & $143(91)$ \\
9-16 years & 134 & $126(94)$ \\
ood donors & 495 & $495(100)$ \\
Cord blood & 702 & $701(100)$ \\
\hline
\end{tabular}

* Number of serum samples evaluated. †Titre of $1: 4$ or greater consi Jered evidence of past CMV infection.

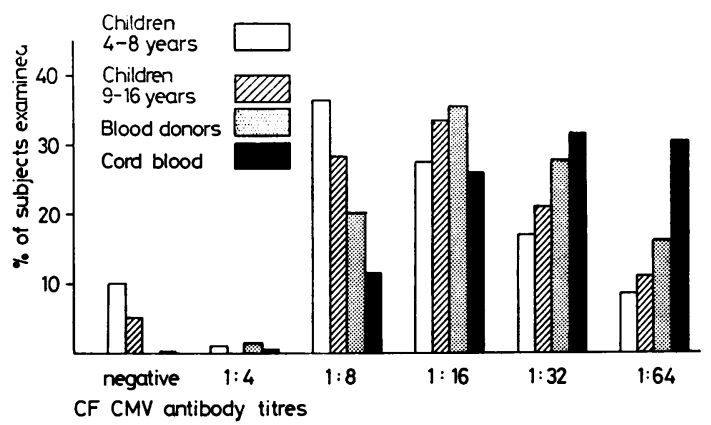

Fig. $1 C F C M V$ antibody titres in different population groups. The highest titres are found in cord blood. The youngest children show the lowest titres (although the incidence of primary infection in this age group is high).

appeared as early as $\mathbf{1 0}$ days or as late as $\mathbf{4 8}$ days after inoculation.

Clinical findings. No apparent malformation, overtly sick child, or dea'th was recorded among the babies examined. There were no differences in the head circumferences $(\mathrm{CMV}+33 \cdot 81 \pm 1 \cdot 54 \mathrm{~cm}, \mathrm{n}=28$; CMV- 33.71 $\pm 1 \cdot 21 \mathrm{~cm}, \mathrm{n}=200$; mean $\pm 1 \mathrm{SD})$ or weights $(\mathrm{CMV}+2995 \pm 455 \mathrm{~g} ; \mathrm{CMV}-3050$ $\pm 418 \mathrm{~g})$ between the infected (CMV+) and 200 randomly selected noninfected (CMV-) infants. Head circumferences $>1$ SD above the mean (36, $36,36$, and $37 \mathrm{~cm})$ were noted in $4(14 \%) \mathrm{CMV}+$ babies, whereas in only $6(3 \%) \mathrm{CMV}-$ babies the same findings were observed (5 with $36 \mathrm{~cm}$, one with $37 \mathrm{~cm}$ head circumference). No microcephaly was noted.

There was an equal distribution of mothers delivering CMV excreters compared with mothers with babies without viruria as far as age and number of pregnancies was concerned.

\section{Discussion}

In this survey of the rate of congenital CMV infection in an African population with a high incidence of CMV infection, measured by the presence of serum CF antibodies, a rate of $1.4 \%$ prenatal CMV infection was found. Urinary CMV excretion within the first 12 hours of delivery was taken as evidence for congenital CMV infection, and the rate of $1.4 \%$ probably somewhat underestimates the true incidence, since failure to isolate the virus owing to nonoptimal conditions of sampling and delay in posting is likely.

A comparison with the findings in Switzerland is illustrated in Fig. 2 (Krech and Jung, 1971; Krech et al., 1971a). It can be seen that there are almost no 


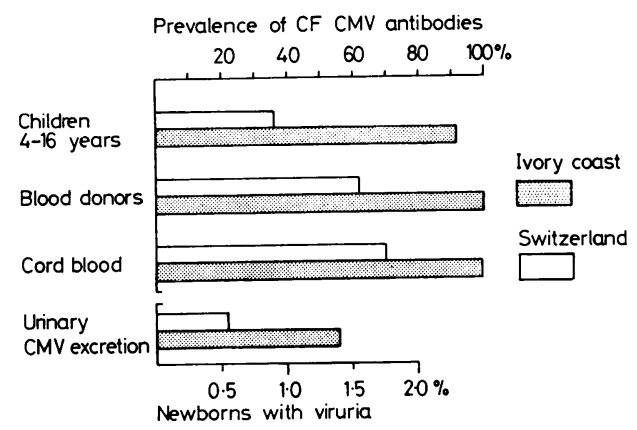

Fig. $2 C F C M V$ antibody prevalence and the role of prenatal $C M V$ infection in Ivory Coast and Switzerland. Prenatal CMV infections are more common in a population (Ivory Coast) where all adults show evidence of past $C M V$ infection.

primary CMV infections in the adult African population as measured by the presence of CF CMV antibodies. Yet, despite the lack of primary CMV infections in adults, the rate of congenital CMV infection is much higher in African than in Swiss babies. These data together with the observations of congenital CMV infections in siblings of consecutive pregnancies (Embil et al., 1970; Krech et al., 1971b; Stagno et al., 1973) and the recent results of Stagno et al. (1977) seem to indicate that the presence of maternal CF antibodies to $\mathrm{CMV}$ does not protect the fetus from becoming infected. Stagno et al. (1977) found that in more than $3.4 \%$ of babies excreting CMV within the first week of delivery, in a selected population, the mothers had been seropositive before pregnancy. Congenital CMV infection does not necessarily produce congenital CMV disease; CMV infection of the fetus in mothers whose primary CMV infection occurred before conception may not be clinically significant (Krech et al., 1971b; Stagno et al., 1973), but long-term follow-up will be needed to determine this question. While many CMV-infected babies are apparently healthy at birth (Starr et al., 1970), late sequelae affecting mainly mental development are observed (Hanshaw et al., 1976) and may be related to subclinical encephalitis after CMV infection in utero. We also cannot rule out the possibility that the slightly increased head circumference in $4(14 \%)$ of 28 congenitally infected babies in this study may be related to CMV disease.

Infection of the fetus in mothers who gave seropositive results could be explained either by reactivation of latent maternal CMV infection during pregnancy, or by reinfection in spite of the presence of serum antibodies. Different strains of CMV might also be responsible for recurrence of infections (Huang et al., 1976). There are, however, only minor antigenic differences between different strains of CMV and cross-immunity occurs (Andersen, 1970; Weller, 1971). Furthermore, genetic and antigenic homology has been demonstrated in CMV strains isolated from prenatally infected babies and their mothers (Stagno et al., 1973, 1977). If primary CMV infection of the mother before pregnancy does not protect the infant from being infected in utero by CMV, then the value of a live CMV vaccine in preventing congenital CMV disease must be questioned (Elek and Stern, 1974). If, on the other hand, the presence of serum antibodies could protect against primary CMV infection, then immunisation with noninfective subunits of CMV, conferring immunity without producin ginfection, may be more promising (Pagano, 1976).

In this survey higher CF CMV antibody titres were observed in women at the end of pregnancy compared with the titres in male blood donors and children (Fig. 1). This observation, at present unexplained, might be related to alterations of virushost interaction during pregnancy. The role of cellmediated immunity in CMV infections also requires further investigation. There is evidence of some impairment of cell-mediated immune mechanisms in mothers delivering prenatally infected babies (Rola-Pleszczynski et al., 1977).

In conclusion, host immunity to CMV infection may be more complex than was previously appreciated. A better understanding of the immune mechanisms may open the way to effective prevention of prenatal CMV infection with its toll of mental retardation (Weller, 1971; Elek and Stern, 1974).

We thank the Ministry of Health of the Government of the Ivory Coast for permission to conduct this survey; all the members of the hospitals of PortBouet and Adjamé and of the Centre de Transfusion in Abidjan for help; the Nestlé Foundation for laboratory facilities, and $\mathrm{Mr}$ Ladji Diaby and $\mathrm{Mr}$ Georges N'Zi for help in organising this study.

\section{References}

Andersen, H. K. (1970). Complement-fixing and virusneutralizing antibodies in cytomegalovirus infection as measured against homologous and heterologous antigen. Acta pathologica et microbiologica Scandinavica (Section B), 78, 504-508.

Elek, S. D., and Stern, H. (1974). Development of a vaccine against mental retardation caused by cytomegalovirus infection in utero. Lancet, 1, 1-5.

Embil, J. A., Ozere, R. L., and Haldane, E. V. (1970). Congenital cytomegalovirus infection in two siblings from consecutive pregnancies. Journal of Pediatrics, 77, 417-421.

Hanshaw, J. B., Scheiner, A. P., Moxley, A. W., Gaev, L., Abel, V., and Scheiner, B. (1976). School failure and deafness after 'silent' congenital cytomegalovirus infection. New England Journal of Medicine, 295, 468-470. 
Huang, E-S., Kilpatrick, B. A.. Huang, Y.T., and Pagano, J. S. (1976). Detection of human cytomegalovirus and analysis of strain variation. Yale Journal of Biology and Medicine, 49, 29-43.

Klein, J. O., Remington, J. S., and Marcy, S. M. (1976). In Infectious Diseases of the Fetus and the Newborn Infant, first edition, pp. 1-32. Edited by J. S. Remington and J. O. Klein. Saunders: Philadelphia.

Krech, U. (1973). Complement-fixing antibodies against cytomegalovirus in different parts of the world. Bulletin of the World Health Organisation, 49, 103-106.

Krech, U., and Jung, M. (1971). Prenatal Infections, pp. 37-43. Edited by O. Thalhammer. Thieme: Stuttgart.

Krech, U., Jung, M., and Jung, F., editors (1971a). Cytomegalovirus Infection of Man, first edition, pp. 88-105. Karger: Basel.

Krech, U. H., Konjajev, Z., and Jung, M. (1971b). Congenital cytomegalovirus infection in siblings from consecutive pregnancies. Helvetica paediatrica acta, 26, 355-362.

Pagano, J. S. (1976). Prevention of infections with cytomegalovirus. Yale Journal of Biology and Medicine, 49, 59-62.

Rola-Pleszczynski, M. R., Frenkel, L. D., Fuccillo, D. A., Hensen, S. A., Vincent, M. R., Reynolds, D. W., Stagno, S., and Bellanti, J. A. (1977). Specific impairment of cellmediated immunity in mothers of infants with congenital infection due to cytomegalovirus. Journal of Infectious Diseases, 135, 386-391.
Stagno, S., Reynolds, D. W., Huang, E-S., Thames, S. D., Smith, R. J., and Alford, C. A., Jr (1977). Congenital cytomegalovirus infection. Occurrence in an immune population. New England Journal of Medicine, 296, 1254-1258.

Stagno, S., Reynolds, D. W., Lakeman, A., Charamella, L. J., and Alford, C. A., Jr (1973). Congenital cytomegalovirus infection: consecutive occurrence due to viruses with similar antigenic compositions. Pediatrics, 52, 788-794.

Starr, J. G., Bart, R. D., Jr, and Gold, E. (1970). Inapparent congenital cytomegalovirus infection: clinical and epidemiologic characteristics in early infancy. New England Journal of Medicine, 282, 1075-1078.

Stern, H., and Tucker, S. M. (1973). Prospective study of cytomegalovirus infection in pregnancy. British Medical Journal, 2, 268-270.

Weller, T. H. (1971). The cytomegalovirus: ubiquitous agents with protean clinical manifestations. New England Journal of Medicine, 285, 203-214.

Correspondence to $\mathrm{Dr} \mathrm{K}$. Schopfer, Section of Immunology, Institute of Medical Microbiology, Frohbergstrasse 3, CH-9000, St Gallen, Switzerland. 